\title{
Über die Fermente des Nucleinstoffwechsels bei der Gicht. Von
}

J. R. Miller und Walter Jones.

(Aus dem physiologisch-chemischen Laboratorium der John Hopkins-Universität.)

(Der Redaktion zugegangen am 12. Juli 1909.)

In einer kürzlich erschienenen Veröffentlichung gab Brugsch ${ }^{1}$ ) eine Übersicht über die Brugsch-Schittenhelmsche Theorie der Gicht. Diese Theorie beruht zum großen Teil auf klinischen Beobachtungen und wird in gewissem Grade durch Experimente mit normalen menschlichen Organen gestützt; sie führt zu der Auffassung, daß die Gicht die Folge einer Störung der Tätigkeit der Fermente des Nucleinstoffwechsels sei, vor allem aber werde sie durch das Fehlen des uricolytischen Ferments, welches die Zerstörung der Harnsäure zur Aufgabe habe, verursacht. Diese Idee wird kaum denjenigen neu erscheinen, die sich mit der Entwicklung der intracellulären Enzyme beschäftigt haben; aber während sie bisher allgemein nur als logische Notwendigkeit aufgefaßt wurde, wird sie hier durch Experimente von nur angenommen direkter Beweiskraft begründet. Wir waren schon lange der Ansicht, daß nur Versuche mit den Organen Gichtkranker einen endgültigen Beweis für die Behauptung Brugsch liefern würden, und haben dauernd nach diesem Material ausgeschaut; vor 3 Monaten starb ein Patient im John Hopkins-Hospital infolge einer chronischen Nephritis und Gicht und die Organe wurden uns zur Untersuchung überlassen. Unsere Bemühungen richteten sich natürlich auf die Frage der Uricolase in der Leber; infolge einer Diskussion, die neulich über dieses Ferment geführt wurde, haben wir uns während der ganzen Dauer des Versuches streng vor dem Ein-

1) Zeitschrift f. Path. u. Therap., Bd. VI, S. 278. 
tritt von Faktoren gehütet, die zu falschen Schlußfolgerungen hätten führen können, entweder durch die Zerstörung des Ferments oder der Harnsäure selbst.

Wir waren leicht in der Lage, uns zu überzeugen, daß die Gichtleber kein Ferment enthielt, welches imstande war, Harnsäure zu zerstören $;^{1}$ ) denn wir fanden nicht nur die Harnsäure wieder, die wir dem wässerigen Extrakt der Leber während einer länger dauernden Digestion bei Körpertemperatur und Luftdurchleitung hinzugefügt hatten, sondern es gelang uns auch, dieselbe selbst in den Fällen unter den Produkten der Autolyse der Leber zu isolieren, in denen wir nicht Harnsäure hinzugefügt hatten. Bei diesem letzten Ergebnis kommt der störende Einfluß des Alkalis, in dem die Harnsäure sonst aufgelöst wird, garnicht in Frage. Diese Bedingungen sind wohl die geeignetsten für den Beweis des Fehlens des Ferments, wenn, wie z. B. hier, ein Gewebe nicht einmal imstande war, die aus seinem eigenen Nucleoproteid entstandene geringe Menge von Harnsäure zu zerstören; es kann auch dann nicht erwartet werden, daß von außen zugefügte Substanz angegriffen wird.

Die Ergebnisse der Arbeiten einer Anzahl yon Forschern scheinen zu dem Schluß zu berechtigen, daß die Uricolase normalerweise in der Leber vorhanden sei; wenn wir nun in unserem Falle zu einem negativen Ergebnis bei der Gichtleber gekommen sind, so wird dieser Nachweis eine Stütze für die Hauptforderung der Brugsch-Schittenhelmschen Hypothese. Unglücklicherweise kommt aber Wiechowski²) in seiner Arbeit in überzeugender Weise zu dem Schluß, daß wässerige Extrakte normaler menschlicher Organe keine zerstörende Wirkung auf Harnsäure ausüben; diese wird bei der Digestion des wässerigen Extraktes der menschlichen Leber selbst unter den günstigsten Bedingungen für die Tätigkeit der Uricolase quantitativ wiedergefunden; dieser Autor wandte bei seiner Arbeit das Gewebe kurz nach dem Tode noch warm an; er konnte aber keinen S. 109.

1) Wiechowski, Hofmeisters Beiträge, Bd. IX, S. 295, Bd. XI,

2) Schmiedebergs Archiv, Bd. LX, S. 197. 
Unterschied entdecken zwischen einem frischen und einem gekochten Extrakt des Organs in seiner Wirkung auf die Harnsäure. Wiechowski konstatiert also, daß seine Ergebnisse im Gegensatz zu den Resultaten der wenigen Autoren stehen, die sich in dieser Frage geäußert haben. So fanden Pfeifer ${ }^{1}$ ) und Croftan ${ }^{2}$ ) die Uricolase in menschlichen Organen. Schittenhelm und Schmid, ${ }^{3}$ ) die nur Teile der kleinen Organe von Kindern untersuchten, fanden nichtsdestoweniger, daß hinzugesetzte Harnsäure verschwindet, auch ohne Luftdurchleitung, vorausgesetzt, daß das Gefäß, in dem die Digestion vor sich geht, häufig geschüttelt wird. Auf Grund des Erscheinens der Wiechowskischen Arbeit beschäftigten wir uns mit der Frage, ob Uricolase in normalen menschlichen Organextrakten vorhanden sei; zu dem Zwecke benutzten wir Lebern von Aneurysmakranken; die Experimente wurden unter verschiedenen Bedingungen gemacht; in einigen Fällen wurde Harnsäure hinzugefügt, in anderen wurden die Produkte der Autodigestion allein untersucht; in fast allen Versuchen wurde Luft durchgeleitet, aber gelegentlich wurde auch das Gefäß ohne Luftdurchleitung geschüttelt; unsere Resultate waren gleichartig; Harnsäure wurde immer gefunden und hinzugefügte Harnsäure konnte fast oder ganz quantitativ wieder aufgefunden werden. Wir können also ohne Zaudern Wiechowskis Schlußfolgerungen unterschreiben: «daß unter den Umständen, unter denen potente tierische Organe Harnsäure restlos zersetzen, von einer analogen Fähigkeit menschlicher Leber nicht die Rede sein kann.» Ferner müssen wir noch hinzufügen, daß ein Unterschied zwischen der Leber von Gicht und Aneurysma in bezug auf Uricolase nicht besteht.

Wir müssen aber noch notwendigerweise die BrugschSchittenhelmsche Hypothese aus einem anderen Grunde in Frage stellen, nämlich mit Rücksicht auf die anderen Fermente des Nucleinstoffwechsels neben der Uricolase; aber vor einer Besprechung des Gichtfalles wird wohl hier ein

1) Hofmeisters Beiträge, Bd. VII, S. 463.

2) Pflügers Archiv, Bd. GXXI, S. 377.

s) Zeitschrift f. Path. u. Ther., Bd. IV, S. 421. 
Wort über die normalen Verhältnisse am Platze sein. Eine Untersuchung über die Nucleinfermente der Organe von Kindern ist von Schittenhelm und Schmid gemacht worden und ihre Ideen werden wohl in den folgenden unzusammenhängenden, ihren Schlußfolgerungen entnommenen Sätzen ausgedrückt: «Wir können ohne weiteres annehmen, daß diese Nuclease sämtlichen Organen in gleicher $\mathrm{W}^{\top}$ eise zukommt ... das desamidierende Ferment, welches genau ebenso verbreitet ist, wie die Nuclease . . . auch dieses Ferment (Xanthinoxydase) scheint sich nahezu überall zu finden, wo Zellkerne sind.» Es wird uns etwas schwierig, den letzten Veröffentlichungen Schittenhelms über dieses Thema zu folgen, da wir so ganz von ihm abweichende Auffassungen über die Fermente selbst haben, daß die Niederschrift des einen dem anderen mehr oder weniger unverständlich erscheint; nach unserer Auffassung (und diese mag vielleicht nicht richtig sein) glaubt Schittenhelm, daß vier und womöglich alle fünf Nucleinfermente in allen Säugetierorganen vorhanden sind ohne Rücksicht auf Spezies und Alter. Bei Besprechung der Fermente des Muskels gebraucht er den Ausdruck «wie es bei der Leber der Fall ist» und bei der Auseinandersetzung über menschliche Organe findet er in der Tat alle diese Fermente in allen Organen. Vom menschlichen Muskel schreibt er: "Aus diesen Untersuchungen folgt klar, daß menschlicher Muskel dieselben Fermente besitzt wie der Muskel der Säuger, also Nuclease, Amidase, Xanthinoxydase und Uricolase.» Schittenhelm hat sich dauernd geweigert, irgend einen Unterschied in den bezüglichen Mengen dieser Fermente zuzugeben (d. h. Schnelligkeit oder Vollständigkeit ihrer Aktion) zum Beweis für ihre Verschiedenheit; wir sind infolgedessen in Verlegenheit zu wissen, ob er glaubt, daß fünf verschiedene Fermente vorliegen oder nur ein Ferment mit fünf verschiedenen Eigenschaften vorhanden ist. Wenn das letztere der Fall ist, dann braucht er nur die Ausdrücke «Nuclease , "Amidase und «Xanthooxydase " aufzugeben und einen Namen für alle drei zu wählen; sonst müßte man denken, daß er eine Ausnahme für die Identität von "Guanase» und *Adenase» macht, während er auf denselben Beweisen sich stützend 
einen Unterschied $z$ wischen "Nuclease und *Xanthooxydase» zuläßt. In dem Bestreben, auf alle Fälle zu beweisen, daß Guanase und Adenase dasselbe Ferment seien, hat sich Schittenhelm die Mühe gemacht, einen bedeutenden Teil unserer Arbeit zu wiederholen, veranlaßt nach seinem Geständnis durch Gründe rein physiologischer Natur; seine Resultate sind nicht immer von unseren eigenen sehr verschieden; in der Tat wir können manche von ihnen mit einem bißchen Zustutzen annehmen; - aber er bringt es immer fertig, einen Schluß zu ziehen, der dem unseren entgegengesetzt ist. Im Interesse der Kürze und der Zeitersparnis würde es für Schittenhelm sehr angebracht sein, uns immer und in allen Dingen zu widersprechen, die wir jemals in der Zukunft veröffentlichen werden.

Es ist unsere Meinung, daß fünf verschiedene Fermente in dem Nucleinstoffwechsel eine Rolle spielen: nämlich Nuclease, Adenase, Guanase, Xanthooxydase und Uricolase. Unser Glaube an die voneinander unabhängige Existenz dieser fünf Fermente, die häufig von einem von uns festgestellt ist, beruht in dem Nachweis ihres verschieden starken Vorhandenseins bei verschiedenen Organen, Spezies und Alter, zwar nicht notwendigerweise auf der vollständigen Abwesenheit des einen von ihnen an einem Ort, obwohl wir bisher nicht imstande waren, auch nur eine Spur von Guanase in der Leber oder Milz des Schweines zu entdecken; von dem letzteren Organ war allerdings Schittenhelm einst so gütig einzugestehen: *geht aber offenbar die Umwandlung des Adenins in Hypoxanthin bei weitem schneller und vollständiger vor sich wie diejenige des Guanins in Xanthin. ${ }^{1}$ )

1) Diese Zeitschrift, Bd. XLVI, S. 365.

Vor kurzem fanden wir in unserem Thermostaten ein Gefäß mit Schweinemilzextrakt vor, dessen Aufschrift lautete: «11. Januar 1903». Wir hatten keine Schwierigkeit, aus diesem Material die übliche Menge von Guanin zu isolieren. Wenn das Extrakt eine Spur von Guanase enthielte, so hätte man wohl erwarten können, daß das Guanin in 6 Jahren verschwunden wäre. Trotz der Abwesenheit von Guanin im Urin vom Schwein und der anatomischen Beziehung der Leber und Milz ist doch die geringste Menge von Guanin in einem Extrakt von Schweinemilz ebenso 
Wir haben in letzter Zeit eine mehr oder weniger vollständige Untersuchung menschlicher Organe von drei verschiedenen Fällen vorgenommen; einer war von einem Typhuskranken, ${ }^{1}$ ) ein zweiter von einer chronischen sclerotischen Endocarditis und ein dritter von einem Aneuryma. Es wurden die Leber, die Milz, die Nieren, Pankreas und die Lunge untersucht. Das gleichmäßige Ergebnis führt ganz klar zu folgenden Schlußfolgerungen:

1. In menschlichen Organextrakten fehlt deutlich die Adenase ganz analog zu dem Fehlen der Guanase in den Organen des Schweines; es ist wohl wahr, daß eine Spur von Hypoxanthin, wie wir glauben, in gewissen Experimenten mit menschlichen Organen gefunden werden kann, aber diese Menge ist so gering, daß die Identität nur durch Sammeln mehrerer Hypoxanthinfraktionen von verschiedenen Experimenten nachgewiesen werden konnte. Übrigens wird diese Spur von Hypoxanthin ebenso wohl bei der Autolyse gefunden, als nach dem Hinzufügen von Adenin und wird nicht größer nach einer Digestion von 25 Tagen als nach 2 Tagen, während das hinzugefügte Adenin sich nicht mit der Zeit vermindert. Der Grund, warum wir nie vorher diese Spur von Hypoxanthin angetroffen haben, ist der, weil wir niemals zuvor (mit einer Ausnahme) ${ }^{2}$ ) eine Drüse untersucht haben, die frei ist von Adenase und Xanthooxydase, eine notwendige Bedingung für das Erscheinen von präformiertem Hypoxanthin. Die Frage über das Vorkommen von Hypoxanthin in verschiedenen Geweben hat keine offenbare Beziehung zu den Fermenten des Nucleinstoffwechsels und wird daher ausführlich in einem Artikel «Präformiertes Hypoxanthin , von V. N. Leonard ${ }^{3}$ ) behandelt werden.

vor Zerfall geschützt wie in einem Präparatenglas. (Schittenhelm und Schmid, Zeitschrift f. Path. u. Therap., Bd. IV, Mendel and Mitchel, Americ. Journ. of Physiol., Bd. XXII, S. 97.)

1) Winternitz und Jones, Diese Zeitschrift, Bd. LX, S. 180.

2) Diese Bedingungen sind bei der Hundeleber erfüllt; wir können uns aber nicht erinnern, ob wir eine Spur von Hypoxanthin gefunden haben.

s) In den folgenden Nummern des Journal of Biological Chemistry, ebenda Schlußfolgerungen $z u$ dem Artikel von Straughn und Jones. 
Es ist uns mit Erfolg gelungen, hinzugefügtes Adenin aus den Extrakten menschlicher Gewebe wiederzugewinnen, und zwar in fast quantitativen Mengen, wie man es unter solchen Versuchsbedingungen erwarten kann.

2. Der hauptsächliche, vielleicht der einzige Ort für die Bildung von Harnsäure (aus Guanin) ist die Leber. Dieses Organ ist reichlich versehen mit beidem: Guanase und Xanthooxydase, so daß verhältnismäßig große Mengen von zugefügtem Guanin vollständig und schnell in Harnsäure oder eine Mischung von Harnsäure und Xanthin umgewandelt werden. Xanthooxydase scheint nur in dieser Drüse vorzukommen; sonst könnte man nicht verstehen, wie auch nur eine Spur von vorgebildetem Hypoxanthin in den anderen Geweben gefunden werden konnte, da doch diese Substanz bei Gegenwart von Xanthooxydase mit der größten Leichtigkeit zu Xanthin oxydiert wird. Die übrigen Drüsen haben keine Tendenz, auch nur den Teil des Xanthins, der vom Guanin stammt, zu Harnsäure zu oxydieren.

Diese teilweise Oxydation von Xanthin geht immer bei Gegenwart von Xanthooxydase selbst vor sich, wenn nicht Luft durchgeleitet wird. Wir unterließen die Durchleitung von Luft in der Erwartung, beides sowohl Harnsäure wie Xanthin zu finden in Übereinstimmung mit den Angaben der anderen Autoren; nimmt man nun die Leber als Vergleichsobjekt, dann enthalten die anderen Gewebe keine Xanthooxydase.

3. Die Milz, die beim Ochsen diese Fermente reichlich enthält, hat beim Menschen weder Guanase noch Adenase.

4. Guanase ist im Überfluß in der Leber, wie festgestellt wurde, und kann auch in der Niere und Lunge vorgefunden werden.

Diese Verteilung der Nucleinfermente in den menschlichen Organen gibt ein charakteristisches und prägnantes Bild, dem sich der Gichtfall sehr wohl anpaßt; man kann bei ihm konstatieren, daß die Verteilung der Nucleinfermente mit dérjenigen der drei übrigen übereinstimmt. Adenase fehlt in allen Organen; die Milz hat keine von beiden Amidasen; Xanthooxydase ist im Überfluß in der Leber vorhanden, aber nur diesem einen Organ vorbehalten; Guanase findet sich in der Leber, Niere 
und Lunge; die konstatierte Spur von Hypoxanthin kann man in verschiedenen Organen vorfinden; es ist sehr zweifelhaft, ob überhaupt die Gicht, wie bis jetzt angenommen wurde, in direkter Beziehung zum Nucleinstoffwechsel steht.

Es ist von uns und von Mendel und Mitchell ${ }^{1}$ ) gezeigt worden, daß beim Tier während der embryonalen Entwicklung eine fortdauernde Bildung der Fermente stattfindet; es ist uns gelungen, dasselbe für den menschlichen Körper zu beweisen $;^{2}$ ) die Leber des Erwachsenen wandelt Guanin schnell und vollständig um, aber es gelang uns, aus einem wässerigen Extrakt der Leber eines 5 Monate alten Foetus das Guanin nach einer Digestion unverändert zurückzugewinnen. Dieses vielleicht stärkste Nucleinferment des erwachsenen Körpers kann nicht im Embryo gefunden werden.

Ein Teil der experimentellen Daten, auf denen die Arbeit beruht, wird im folgenden gegeben; die Methoden sind so bekannt und so oft wiederholt, daß sie nicht erst angeführt wurden.

1. Foetus 5 Monate alt: wässeriges Leberextrakt (1:4) $60 \mathrm{ccm}$, Guaninsulfat $150 \mathrm{mg}$, entsprechend Guanin $105 \mathrm{mg}$; digeriert bei $40^{\circ} 4$ Tage.

Gefunden: $84 \mathrm{mg}$ Guanin oder 80\%, kein Xanthin.

2. Fall von Aneurysma: wässeriges Leberextrakt (1:4) $500 \mathrm{ccm}$, Harnsäure $400 \mathrm{mg}$; digeriert bei $40^{\circ} 3$ Tage, Luft wurde in Zwischenräumen 12 Stunden durchgeleitet.

Gefunden: $420 \mathrm{mg}$ Harnsäure.

3. Derselbe Fall: wässeriges Leberextrakt $1000 \mathrm{ccm}$, ohne Zusatz von Harnsäure; digeriert 3 Tage bei $40^{\circ}$, Luftdurchleitung in Zwischenräumen während 11 Stunden.

Gefunden: $105 \mathrm{mg}$ Harnsäure.

4. Fall von chronischer Nephritis und Gicht: Leberextrakt $350 \mathrm{ccm}$, Harnsäure $300 \mathrm{mg}$; digeriert 3 Tage bei $40^{\circ}$, Luftdurchleitung in Zwischenräumen während 9 Stunden.

Gefunden: 320 mg Harnsäure.

1) loc. cit.

2) Winternitz und Jones, loc. cit. 
5. Derselbe Fall: wässeriges Milzextrakt $200 \mathrm{ccm}$, Adeninsulfat $200 \mathrm{mg}$, entsprechend Adenin $133 \mathrm{mg}$; digeriert bei $40^{\circ}$ 25 Tage.

Gefunden: 349 mg Adeninpikrat, entsprechend $122 \mathrm{mg}$ Adenin; wiedergewonnen $92 \%$.

6. Derselbe Fall; wässeriges Milzextrakt $200 \mathrm{ccm}$, Guaninsulfat $200 \mathrm{mg}$, entsprechend Guanin $140 \mathrm{mg}$; digeriert 4 Tage. Gefunden $123 \mathrm{mg}$ Guanin, Ausbeute $88 \%$; kein Xanthin.

7. Derselbe Fall: wässeriges Pankreasextrakt $250 \mathrm{ccm}$, Adeninsulfat $250 \mathrm{mg}$, entsprechend $167 \mathrm{mg}$ Adenin; digeriert 4 Tage bei $40^{\circ}$.

Gefunden: 398 mg Adeninpikrat, entsprechend 139 mg Adenin. Ausbeute $83 \%$.

8. Derselbe Fall: wässeriges Leberextrakt $500 \mathrm{ccm}$, Adeninsulfat $300 \mathrm{mg}$, entsprechend $200 \mathrm{mg}$ Adenin; digeriert 25 Tage. Gefunden: $530 \mathrm{mg}$ Adeninpikrat, entsprechend $185 \mathrm{mg}$ Adenin.

Ausbeute $93 \%$. Harnsäure $42 \mathrm{mg}$; Xanthin eine Spur.

9. Derselbe Fall: wässeriges Leberextrakt $500 \mathrm{ccm}$, Guaninsulfat $350 \mathrm{mg}$, entsprechend $245 \mathrm{mg}$ Guanin; digeriert 2 Tage. Gefunden: Harnsäure $160 \mathrm{mg}$, Xanthin $105 \mathrm{mg}$, kein Guanin.

10. Derselbe Fall; wässeriges Nierenextrakt $250 \mathrm{ccm}$, Adeninsulfat $200 \mathrm{mg}$, entsprechend $133 \mathrm{mg}$ Adenin; 5 Tage digeriert.

Gefunden: $301 \mathrm{mg}$ Adeninpikrat, entsprechend $105 \mathrm{mg}$ Adenin.

$$
\text { Ausbente } 80 \% \text {. }
$$

11. Derselbe Fall: wässeriges Extrakt der Niere $250 \mathrm{ccm}$, Guaninsulfat $200 \mathrm{mg}$, entsprechend Guanin $140 \mathrm{mg}$; digeriert 8 Tage.

Gefunden: Xanthin $114 \mathrm{mg}$, entsprechend $82 \%$ Guanin; Guanin nicht mehr vorhanden.

12. Endocarditis : wässeriges Nierenextrakt $250 \mathrm{ccm}$, Adeninsulfat $200 \mathrm{mg}$, entsprechend $103 \mathrm{mg}$ Adenin; digeriert 25 Tage. Gefunden: 307 mg Adeninpikrat, entsprechend 137 mg Adenin.

$$
\text { Ausbeute } 81 \% \text {. }
$$

13. Derselbe Fall: wässeriges Nierenextrakt $250 \mathrm{ccm}$, Guaninsulfat $200 \mathrm{mg}$, entsprechend $140 \mathrm{mg}$ Guanin; digeriert 10 Tage. 
404 J. R. Miller u. W. Jones, Über Fermente des Nucleinstoffwechsels.

Gefunden: $118 \mathrm{mg}$ Xanthin oder 84\% Guanin; kein Guanin mehr.

14. Derselbe Fall: wässeriges Extrakt der Lunge $300 \mathrm{ccm}$, Adeninsulfat $300 \mathrm{mg}$. entsprechend $200 \mathrm{mg}$ Adenin, digeriert 3 Tage.

Gefunden: Adeninpikrat $492 \mathrm{mg}$, entsprechend $172 \mathrm{mg}$ Adenin. Ausbeute $86 \%$.

15. Derselbe Fall: wässeriges Extrakt der Lunge $300 \mathrm{ccm}$, Guaninsulfat $300 \mathrm{mg}$, entsprechend $210 \mathrm{mg}$ Guanin; digeriert 2 Tage.

Gefunden: $175 \mathrm{mg}$ Xanthin oder 83\% Guanin; kein Guanin. 16. Derselbe Fall: wässeriges Milzextrakt $200 \mathrm{ccm}$, Adeninsulfat $200 \mathrm{mg}$, entsprechend $133 \mathrm{mg}$ Adenin; digeriert 5 Tage. Gefunden: $349 \mathrm{mg}$ Adeninpikrat, entsprechend $122 \mathrm{mg}$ Adenin. Ausbeute $92 \%$.

17. Derselbe Fall: wässeriges Nierenextrakt $250 \mathrm{ccm}$, Adeninsulfat $200 \mathrm{mg}$, Adenin $133 \mathrm{mg}$; digeriert 20 Tage. Gefunden: $291 \mathrm{mg}$ Adeninpikrat, entsprechend $102 \mathrm{mg}$ Adenin. Ausbeute $76 \%$. 